\title{
Eficiência de uso da radiação fotossinteticamente ativa pela cultura do tomateiro em diferentes ambientes(1)
}

\author{
Bernadete Radin ${ }^{(2)}$, Homero Bergamaschi(3), Carlos Reisser Junior(4), Nídio Antonio Barni(2), \\ Ronaldo Matzenauer(2) e Ivo Antônio Didoné(2)
}

\begin{abstract}
Resumo - A produção de biomassa pelas culturas está relacionada à quantidade de radiação fotossinteticamente ativa interceptada e absorvida pelas folhas, bem como à eficiência com que estas convertem a energia radiante em energia química, pela fotossíntese. O objetivo deste trabalho foi avaliar a eficiência de uso da radiação fotossinteticamente ativa pelo tomateiro (Lycopersicon esculentum Mill.) cultivado em diferentes ambientes. Os experimentos foram realizados em estufa de plástico com e sem tela lateral antiinsetos e fora da estufa, em duas épocas (primavera-verão e verão-outono), no ano agrícola de 1999/2000. Mediu-se a matéria seca aérea e o índice de área foliar ao longo dos dois ciclos, assim como os fluxos de radiação incidente e transmitida. O ambiente em estufa com tela lateral antiinsetos teve menos radiação incidente e maior eficiência de seu uso: 0,44 e $0,60 \mathrm{~g}$ de matéria seca $\mathrm{mol}^{-1}$, nas primeira e segunda épocas, respectivamente. No ambiente fora da estufa, com mais radiação incidente, houve menor eficiência de seu uso $\left(0,30\right.$ e $\left.0,32 \mathrm{~g} \mathrm{~mol}^{-1}\right)$, enquanto no ambiente em estufa sem tela lateral antiinsetos, foram obtidos valores intermediários de eficiência de uso da radiação $\left(0,45\right.$ e $\left.0,53 \mathrm{~g} \mathrm{~mol}^{-1}\right)$.
\end{abstract}

Termos para indexação: Lycopersicon esculentum, radiação solar, estufa, cultivo protegido.

\section{Use efficiency of photosynthetically active radiation by tomato plants grown in different environments}

\begin{abstract}
Crop biomass production is related to the amount of photosynthetically active radiation intercepted and absorbed by the leaves, as well as to their efficiency of conversion of this radiant energy into chemical energy through photosynthesis. The objective of this study was to evaluate the radiation use efficiency by tomato plants (Lycopersicon esculentum Mill.) grown in different environments. Experiments were carried out in plastic-covered greenhouses with and without antiinsects screens and at open air plots, in different growth periods (spring-summer and summer-autumn) during the 1999/2000 crop season. Measurements of dry above-ground biomass and leaf area index throughout both crop cycles were performed, and the incident and transmitted radiation fluxes were registered. The greenhouse with antiinsects screens had less incident radiation, but resulted in higher use efficiency: 0.44 and $0.60 \mathrm{~g}$ dry matter $\mathrm{mol}^{-1}$ during the first and second cycles, respectively. Outside the greenhouses, there was a higher amount of incident radiation, however a lower use efficiency $(0.30$ and $0.32 \mathrm{~g} \mathrm{~mol}^{-1}$ for the first and second cycles, respectively), while the greenhouse without antiinsects screens had intermediate values $\left(0.45\right.$ and $\left.0.53 \mathrm{~g} \mathrm{~mol}^{-1}\right)$.
\end{abstract}

Index terms: Lycopersicon esculentum, solar radiation, greenhouses, protected cultivation.

\footnotetext{
(1) Aceito para publicação em 8 de julho de 2003.

Extraído da tese de doutorado apresentada pelo primeiro autor à Universidade Federal do Rio Grande do Sul (UFGRS), Porto Alegre, RS.

(2)Fundação Estadual de Pesquisa Agropecuária, Rua Gonçalves Dias, 570, Bairro Menino Deus, CEP 90130-060 Porto Alegre, RS. E-mail: bernadete-radin@ fepagro.rs.gov.br,
}

nidio-barni@fepagro.rs.gov.br, ronaldo-matzenauer@fepagro.rs.gov.br, ivo-didoné@ fepagro.rs.gov.br

(3)UFGRS, Caixa Postal 776, CEP 91501-970 Porto Alegre, RS. E-mail: homerobe@ufrgs.br

${ }^{(4)}$ Embrapa-Centro de Pesquisa Agropecuária de Clima Temperado, Caixa Postal 403, CEP 96001-970 Pelotas, RS. E-mail: reisser@cpact.embrapa.br 


\section{Introdução}

A relação existente entre a produção de matéria seca e a quantidade de radiação fotossinteticamente ativa (RFA) interceptada ou absorvida tem sido amplamente usada para definir a eficiência de uso da radiação pelas culturas (Sivakumar \& Virmani, 1984; Costa et al., 1996). Considera-se que, em plantas sadias adequadamente supridas de água e nutrientes, a fotossíntese líquida e a produção de fitomassa sejam proporcionais à quantidade de RFA absorvida pelo dossel (Monteith, 1977). Entretanto, cuidados são necessários quando se compara a produtividade da cultura em diferentes níveis de radiação (Russell et al., 1989), pois a taxa fotossintética e a densidade de fluxo de radiação não têm relação linear dentro do dossel, já que a maioria das folhas está exposta a baixos níveis de radiação.

A absorção da radiação incidente pelas culturas depende do seu índice de área foliar (IAF), posição solar, geometria e tamanho da folha, ângulo de distribuição, idade, arranjo das plantas, época do ano e nebulosidade (Varlet-Grancher et al., 1989) e ainda da espécie cultivada, das condições meteorológicas e de práticas de manejo da cultura. Gallo et al. (1993) mostraram que a eficiência de uso da radiação pode variar, dependendo apenas de como a matéria seca (aérea ou total) e a radiação solar (incidente, interceptada ou absorvida) são definidas e medidas.

A eficiência de conversão decresce gradualmente com o aumento do fluxo de radiação solar acima do ótimo, podendo ocorrer saturação das folhas superiores. Entretanto, o nível de radiação em que ocorre a saturação nas folhas localizadas no interior do dossel é muito mais elevado, pois a incidência de radiação é menor, e muitas das folhas podem não chegar à saturação (Russell et al., 1989). Sinclair \& Horie (1989) verificaram que, em baixo IAF, a eficiência de uso da radiação é baixa, pois a maioria das folhas está sujeita à saturação fotossintética por radiação. Como a fração de área foliar sombreada aumenta com o decorrer do ciclo da cultura, aumenta também a eficiência de uso da radiação. Isto decorre do incremento na contribuição relativa das folhas sombreadas para o acúmulo de biomassa da cultura, à medida que aumenta a fração difusa, e também da maior uniformidade da radiação no interior do dossel.
Os valores de eficiência do uso da radiação em ambiente protegido têm sido maiores do que os encontrados no campo, por causa do aumento da radiação difusa (Hammer \& Vanderlip, 1989; Sinclair et al., 1992). Através de modelos, Sinclair et al. (1992) encontraram que um aumento na fração difusa resultou em maior eficiência de uso da radiação na cultura do milho e da soja.

O objetivo deste trabalho foi avaliar a eficiência do uso da radiação fotossinteticamente ativa pelo tomateiro cultivado em diferentes ambientes.

\section{Material e Métodos}

Os experimentos foram realizados na Fundação Estadual de Pesquisa Agropecuária - Fepagro Saúde Animal, no Município de Eldorado do Sul, RS (latitude 305' S; longitude $51^{\circ} 39^{\prime} \mathrm{W}$; altitude $10 \mathrm{~m}$ ), de setembro de 1999 a junho de 2000. O clima da região é subtropical úmido de verão quente, do tipo fundamental $\mathrm{Cfa}$, conforme a classificação de Köppen. Foi utilizada a cultivar de tomateiro Floradade, americana, de hábito de crescimento determinado, do tipo salada. A semeadura foi realizada em substrato à base de compostagem de pinus, nos dias 8/9/1999 e 19/1/2000. O transplante para os locais definitivos foi efetuado em 18/10/1999 (primeira época) e em 15/2/2000 (segunda época).

Foi utilizada uma estufa de plástico, coberta com polietileno transparente de baixa densidade, com área de $240 \mathrm{~m}^{2}$, pé direito lateral de $3,0 \mathrm{~m}$ e altura máxima de $4,5 \mathrm{~m}$ na parte central. A estufa foi dividida em dois ambientes: com tela antiinsetos (CT) e sem tela (ST), além do ambiente externo à estufa (FE). Cada ambiente continha cinco canteiros com 1,60 $\mathrm{m}$ de largura e espaçamento de $0,40 \mathrm{~m}$ entre eles, com arranjo em linhas pareadas, com espaçamento de $0,80 \mathrm{~m}$ no canteiro e 1,20 m entre as linhas de canteiros vizinhos, e $0,50 \mathrm{~m}$ na linha. Com este arranjo, a população era de duas plantas por metro quadrado.

Como a cultivar utilizada é de crescimento determinado, realizou-se uma única poda para a retirada dos brotos abaixo do primeiro rácimo, a partir de 20 dias após o transplante. As plantas foram tutoradas para impedir o tombamento e permitir maior penetração de radiação solar e ventilação dentro do dossel. A radiação solar global (Rs) e a RFA foram medidas por sensores acoplados a um "datalogger" (modelo CR10X, Campbell Scientific, Logan, EUA). As leituras eram feitas a cada dez segundos e seus valores médios eram registrados a cada 30 minutos. Como houve problemas no registro, em alguns dias, 
foram incluídos dados da estação agrometeorológica do Instituto Rio-Grandense do Arroz (Irga), do Município de

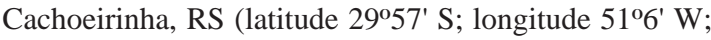
altitude de $13 \mathrm{~m}$ ). Para estimar os dados que faltavam, ajustou-se uma função linear entre os dados de radiação solar global coletados no local do experimento e na estação do Irga, obtendo-se a seguinte equação:

$\mathrm{y}=1,1568 \mathrm{x}, \mathrm{R}^{2}=0,883^{*}$

em que y é o valor de radiação solar global estimado para um determinado dia e x é o dado de entrada da Rs medido na estação do Irga.

Um piranômetro (LI-COR), instalado na parte superior externa da estufa, registrava a Rs, e três sensores "quantum" da mesma marca, que registravam a RFA, foram instalados a $2 \mathrm{~m}$ de altura, no centro de cada ambiente. Também havia três barras providas com sete células de silício amorfo, ligadas em paralelo e com espaços de $15 \mathrm{~cm}$, posicionadas a $10 \mathrm{~cm}$ da superfície do solo, voltadas para cima, para medir a RFA transmitida. Cada barra foi calibrada utilizando um sensor "quantum" de referência. Os dados de RFA foram registrados em $\mu \mathrm{mol} \mathrm{s}^{-1} \mathrm{~m}^{-2}$ e convertidos em mol m${ }^{-2} \mathrm{~s}^{-1}$, adotando o seguinte procedimento:

$\mathrm{RFA}=\sum_{\text {diário }} \frac{\left(\mathrm{RFA}\left(\mu \mathrm{mol} \mathrm{s} \mathrm{s}^{-1}\right) \times \mathrm{t}(\mathrm{s})\right.}{1.000 .000}\left(\right.$ mol de fótons $\left.\mathrm{m}^{-2} \mathrm{dia}^{-1}\right)$.

Para fins de comparação, os dados foram transformados em $\mathrm{MJ} \mathrm{m}^{-2} \mathrm{dia}^{-1}$, utilizando-se um único valor de conversão para os diferentes ambientes, conforme Thimijan \& Heins (1983), pela seguinte fórmula:

$\mathrm{RFA}=\sum_{\text {diário }} \frac{\left(\mathrm{RFA}\left(\mu \mathrm{mol} \mathrm{s}^{-1} \mathrm{~m}^{-2}\right) \times \mathrm{t}(\mathrm{s}) / 4,57\right.}{1.000 .000}\left(\mathrm{MJ} \mathrm{m}^{-2} \mathrm{dia}^{-1}\right)$,

em que RFA é a radiação fotossinteticamente ativa, t é o tempo entre as coletas, e 4,57 é o valor de conversão. Todos os valores calculados a cada 30 minutos foram somados.

A razão RFA/Rs, utilizando-se a radiação global em $\mathrm{MJ} \mathrm{m^{-2 }}$ dia $^{-1}$ e a radiação fotossinteticamente ativa em mol m${ }^{-2} \mathrm{dia}^{-1}$, foi de 1,66, 1,10 e 1,19 nos ambientes FE, CT e ST, respectivamente. Na falta de dados de RFA, as falhas foram preenchidas estimando-a em função da Rs. A partir da primeira semana após o transplante, coletaramse três plantas por ambiente, com amostragens semanais (primeira época) e quinzenais (segunda época). A área foliar foi medida por planímetro ótico modelo LI 3000 (LICOR, Lincon, EUA), e após calculou-se o IAF pela seguinte fórmula:

$\mathrm{IAF}=\mathrm{AF} / \mathrm{S}$,

em que $\mathrm{AF}$ é a área foliar $\left(\mathrm{m}^{2}\right)$ e $\mathrm{S}$ é a área do terreno ocupada pela amostra $\left(\mathrm{m}^{2}\right)$.

A radiação fotossinteticamente ativa interceptada $\left(\mathrm{RFA}_{\text {int }}\right)$ pela cultura foi calculada da seguinte maneira (Varlet-Grancher et al., 1989):
$\mathrm{RFA}_{\text {int }}=\mathrm{RFA}_{\text {inc }}-\mathrm{RFA}_{\mathrm{ts}}$,

em que $\mathrm{RFA}_{\text {inc }}$ é a radiação incidente e $\mathrm{RFA}_{\mathrm{ts}}$ é a radiação transmitida ao solo. A eficiência de intercepção $\left(\varepsilon_{\text {int }}\right)$ da RFA pela cultura foi estimada considerando-se a $R_{F A} A_{\text {int }} \mathrm{e}$ a RFA inc pela expressão:

$\varepsilon_{\text {int }}=\frac{\mathrm{RFA}_{\text {int }}}{\mathrm{RFA}_{\text {inc }}}$.

Um modelo exponencial foi ajustado para estimar a $\varepsilon_{\text {int }}$ em função do IAF, com base em dados de IAF obtidos nas amostragens e na $\varepsilon_{\text {int }}$ média dos dias anteriores e posteriores às amostragens.

O total de massa de matéria seca da parte aérea $\left(\mathrm{g} \mathrm{m}^{-2}\right)$ se ajustou a um modelo linear, em função da RFA interceptada acumulada. $\mathrm{O}$ coeficiente angular da equação representa a eficiência do uso da RFA interceptada.

\section{Resultados e Discussão}

O fluxo da radiação solar global (Rs) foi maior no período primavera-verão, com média de $22,74 \mathrm{MJ} \mathrm{m}^{-2}$ dia $^{-1}$, do que no verão-outono, em que a média ficou em 14,61 $\mathrm{MJ} \mathrm{m}^{-2} \mathrm{dia}^{-1}$ e a RFA inc $_{\text {apre- }}$ sentou comportamento similar ao da radiação solar global, porém com valores inferiores (Figura 1).

$\mathrm{O}$ índice de área foliar (IAF) sempre foi maior nos ambientes CT e ST e menor no ambiente FE (Figura 2). Em geral, folhas que crescem em menor disponibilidade de radiação têm maior superfície e são mais finas do que folhas que crescem expostas ao sol. Estas são mais espessas, pois formam células paliçádicas maiores ou mais camadas dessas células (Boardman, 1977). Outro fator que pode estar envolvido na diferença entre o IAF dos ambientes é a umidade relativa do ar (UR). Nos ambientes de estufa a UR foi maior. Com isso, o potencial da água na folha tende a ser maior, o que pode explicar a maior área foliar nesses ambientes (Gislerod \& Mortensen, 1991).

À medida que aumenta o IAF, a $\varepsilon_{\text {int }}$ da RFA aumenta exponencialmente (Figura 3). A inclinação da curva de $\varepsilon_{\text {int }}$, em IAF baixo, foi maior no ambiente FE e menos acentuada nos ambientes ST e CT. Nestes, foi necessário maior IAF para uma mesma $\varepsilon_{\text {int }}$, o que pode ser atribuído à maior disponibilidade de radiação difusa. Apesar de não ter sido medida a radiação difusa neste trabalho, vários autores já de- 
monstraram que ela é maior dentro de estufas de plástico do que em ambiente externo. Farias et al. (1993) e Camacho et al. (1995) verificaram que a radiação difusa ocorrida externamente foi inferior, na maior parte do período, sendo, em média, $65 \%$ daquela observada no interior da estufa de plástico.
No ambiente FE e com IAF ao redor de 3, a cultura do tomateiro chegou próximo de sua eficiência máxima $(0,939)$ pelo modelo ajustado; no ambiente ST, com IAF maior do que 3 , a $\varepsilon_{\text {int }}$ chegou próximo à máxima eficiência $(0,818)$, e no ambiente $\mathrm{CT}$, a $\varepsilon_{\text {int }}$ chegou próximo à máxima $(0,957)$ em maiores IAF
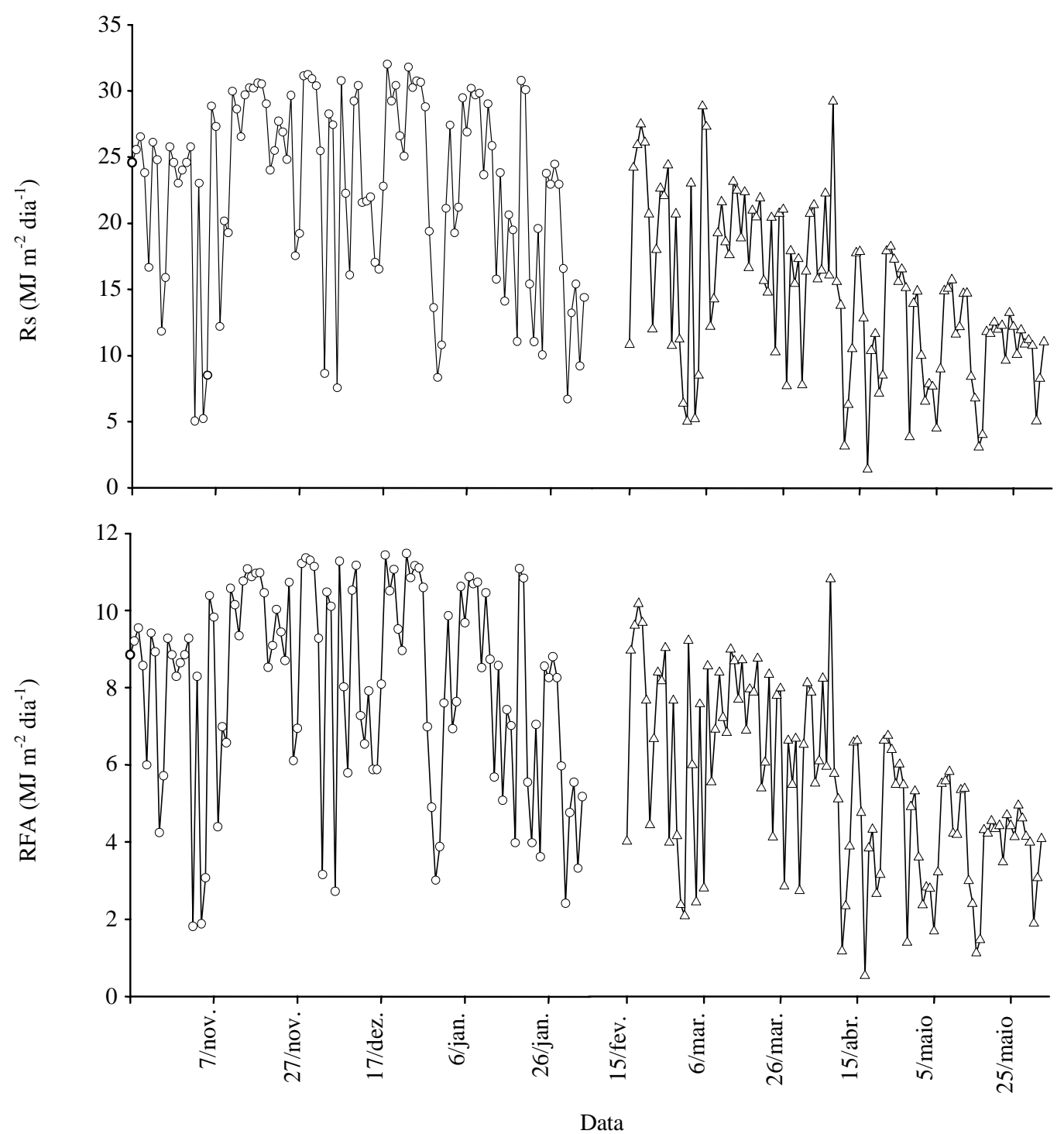

Figura 1. Radiação solar global incidente (Rs) e radiação fotossinteticamente ativa incidente (RFA) na parte externa à estufa, durante dois ciclos da cultura do tomateiro. Eldorado do Sul, RS, 18/10/1999 a 3/2/2000 e $15 / 2$ a 2/6/2000. 
observados. A $\varepsilon_{\text {int }}$ da RFA apresentou aumentos decrescentes com o crescimento da área foliar para todo o conjunto de dados, chegando próximo à eficiência máxima de intercepção $(0,876)$ em maiores IAF (Figura 4), ajustando-se ao modelo de intercepção utilizado por Russell et al. (1989) e Müller (2001).

A eficiência de uso da RFA no ambiente FE foi de 0,30 e 0,32 g de matéria seca (MS) mol$^{-1}(1,39$ e $1,46 \mathrm{~g} \mathrm{MJ}^{-1}$ ) de $\mathrm{RFA}_{\text {int }}$ nas primeira e segunda épocas, respectivamente. No ambiente ST, os valores foram de 0,45 e 0,53 $\mathrm{g} \mathrm{MS} \mathrm{mol}^{-1}$ (2,06 e 2,43 $\left.\mathrm{g} \mathrm{MJ}^{-1}\right)$, e no ambiente $\mathrm{CT}$ a eficiência de uso da radiação foi de 0,44 e $0,60 \mathrm{~g} \mathrm{MS} \mathrm{mol}^{-1}$ (2,00 e 2,72 $\mathrm{g} \mathrm{MJ}^{-1}$ ) nas primeira e segunda épocas, respectivamente (Figura 5).
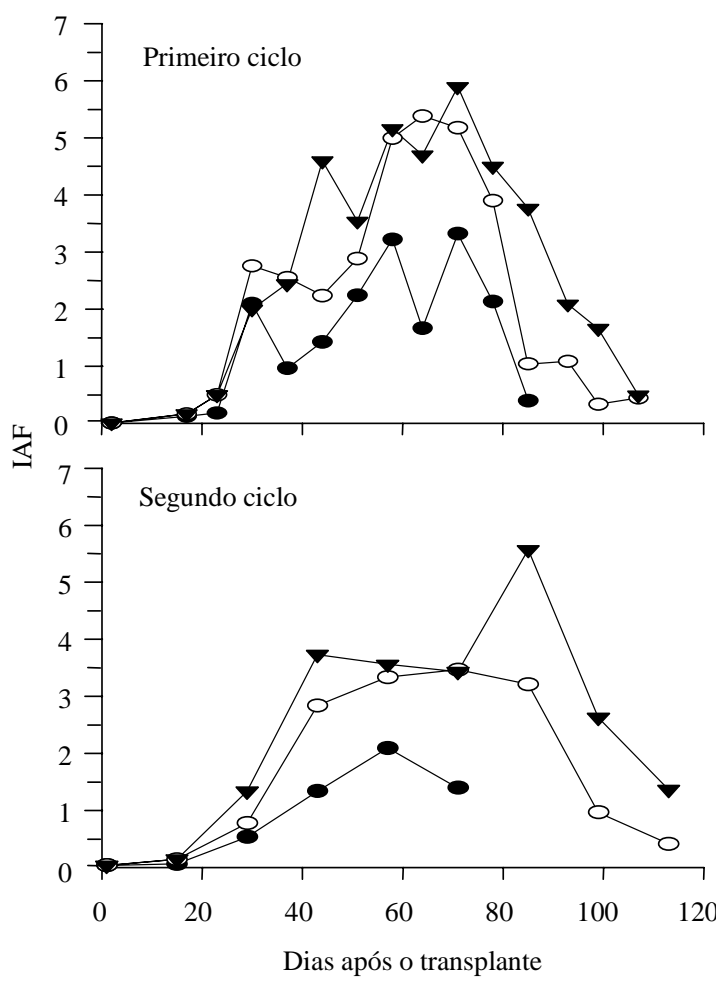

Figura 2. Índice de área foliar (IAF) durante dois ciclos da cultura do tomateiro, em estufa com tela $(\boldsymbol{\nabla})$, sem tela $(\bullet)$ e em ambiente externo à estufa (O). Eldorado do Sul, RS, 1999/2000.
Em relação à cultura do tomateiro em estufa, Heuvelink (1995) também encontrou valor médio de eficiência do uso de 2,5 $\mathrm{g} \mathrm{MS} \mathrm{MJ-1}^{-1}$ de RFA incidente, empregando uma densidade de 2,1 plantas $\mathrm{m}^{-2}$. Andriolo \& Falcão (2000) encontraram valores de 1,60 g MS MJ-1 de RFA. Essas diferenças podem ser atribuídas a diferenças na den-
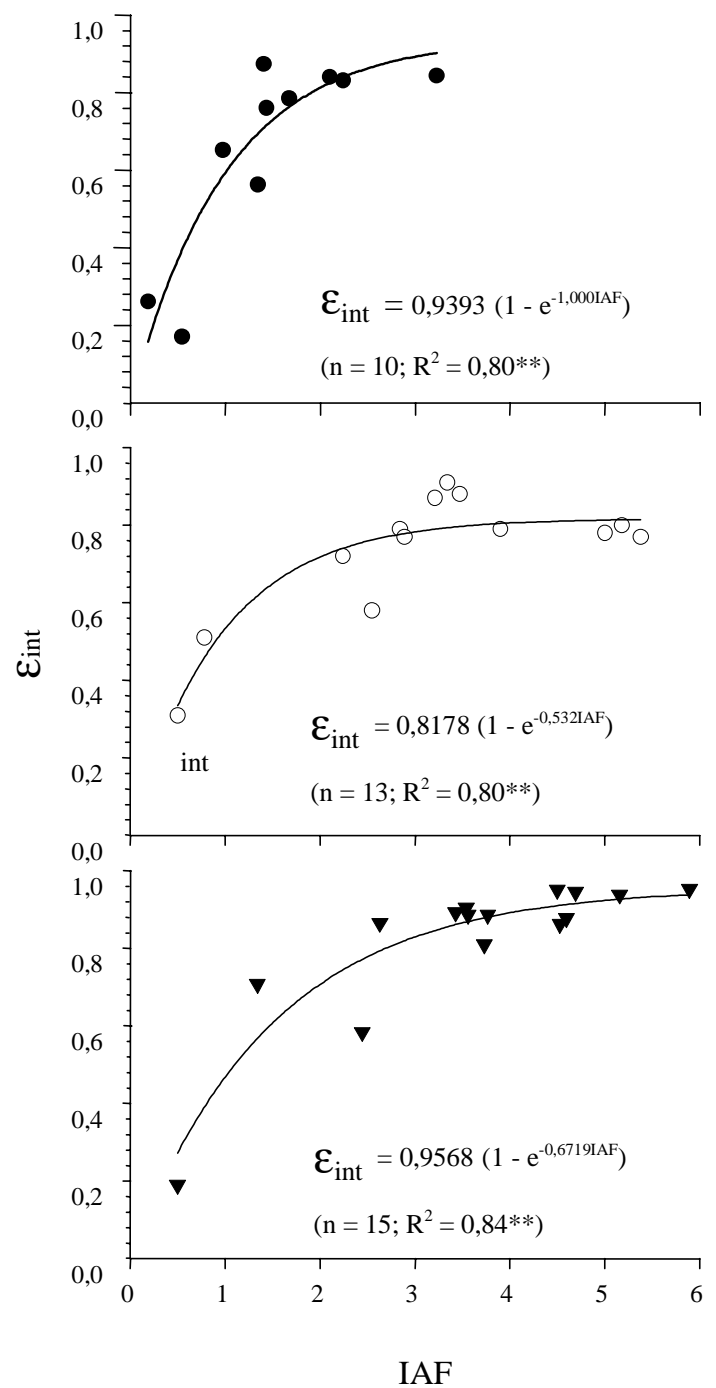

Figura 3. Eficiência de intercepção da radiação fotossinteticamente ativa $\left(\varepsilon_{\text {int }}\right)$ observada em tomateiro em razão do índice de área foliar (IAF), com dados dos primeiro e segundo ciclos, em estufa com tela $(\boldsymbol{\nabla})$, sem tela (O) e em ambiente externo (•). Eldorado do Sul, RS, 1999/ 2000.

Pesq. agropec. bras., Brasília, v. 38, n. 9, p. 1017-1023, set. 2003 
sidade da população de plantas, hábito de crescimento da cultura, cultivares, sistema de poda, desbaste foliar e de como foram tomados os valores de matéria seca (total ou aérea) e a RFA (interceptada, absorvida ou incidente).

No ambiente FE, com maior quantidade de RFA incidente, a biomassa produzida foi menor, ao contrário do verificado em CT e ST em que, com menor disponibilidade de radiação solar, ocorreu maior produção de matéria seca nos dois ciclos de desenvolvimento da cultura. Papadopoulos \& Ormrod (1988) e Hammer \& Vanderlip (1989) obtiveram resultados semelhantes e consideraram que a maior eficiência de uso da radiação em estufas é explicada pelo fato de que, nesses ambientes, existe aumento da radiação difusa. Aikman (1989) constatou que o aumento da radiação difusa promoveu maior uniformidade da radiação no interior do dossel, fazendo com que as folhas inferiores aumentassem a $\varepsilon_{\text {int }}$ e o uso da radiação. Com isso, maior eficiência de uso da radiação pode ocorrer por causa do aumento da contribuição relativa das folhas sombreadas para o acúmulo de biomassa da cultura, o qual cresce à medida que aumenta a fração difusa.

Sinclair \& Horie (1989) constataram que a eficiência de uso da radiação varia dentro de uma mesma

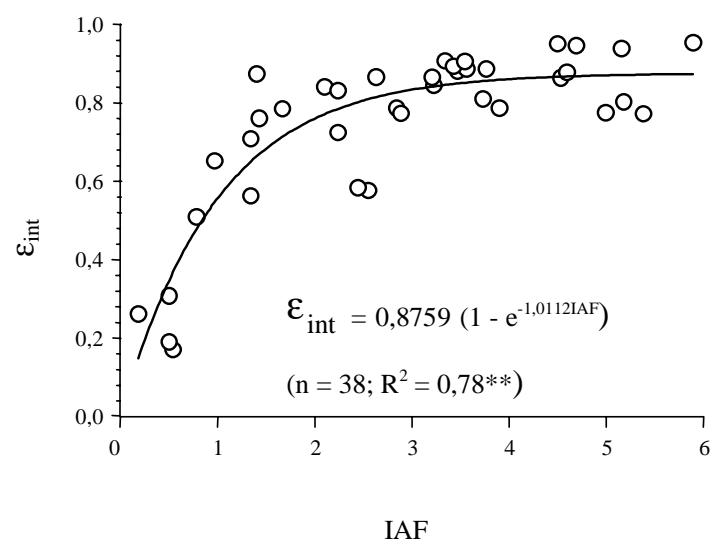

Figura 4. Eficiência de intercepção da radiação fotossinteticamente ativa $\left(\varepsilon_{\text {int }}\right)$ em tomateiro, para todo o conjunto de dados das duas épocas, em estufa com tela, estufa sem tela e em ambiente externo em razão do índice de área foliar (IAF). Eldorado do Sul, RS, 1999/2000. espécie e que folhas saturadas por radiação são menos eficientes do que as sombreadas. A distribuição mais homogênea da radiação solar através do dossel tende a não saturar a maioria das folhas, justificando-se a resposta linear de acúmulo de matéria seca em função do total de RFA interceptada (Russell et al., 1989). No presente trabalho, a eficiência de uso da $\mathrm{RFA}_{\text {int }}$ foi menor durante o período primavera-verão do que verão-outono. Sinclair \& Shiraiwa (1993) também obtiveram maior eficiência de uso da radiação em local onde a média diária de radiação foi menor.

Na média das duas épocas de cultivo, a eficiência de uso da radiação fotossinteticamente ativa foi maior nos ambientes CT e ST do que no ambiente FE. A presença de tela propiciou distribuição mais uniforme da radiação dentro do dossel vegetal, favorecendo a expansão foliar e, conseqüentemente, a eficiência de uso da radiação.
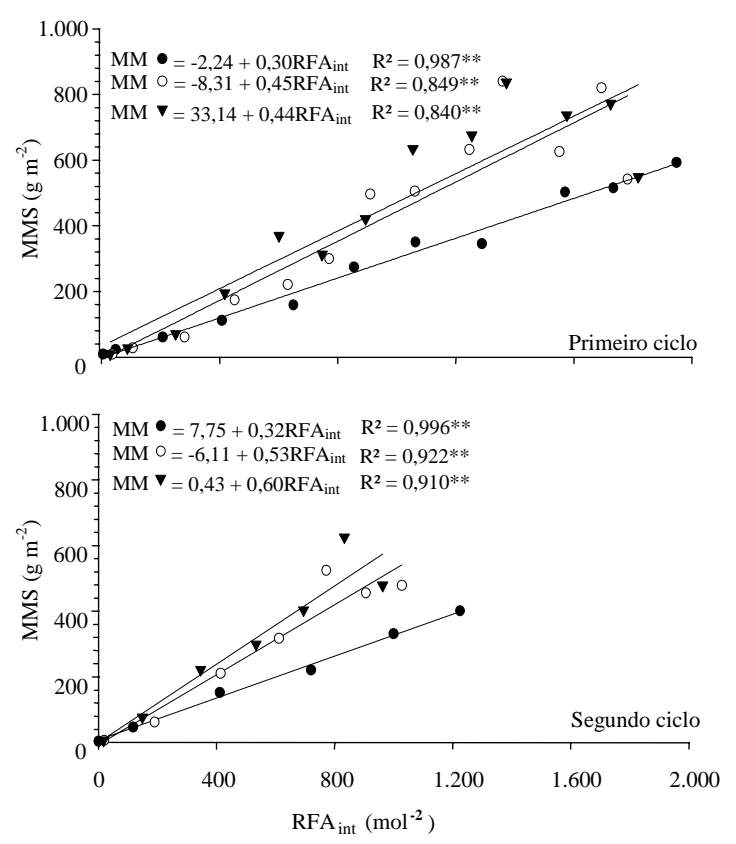

Figura 5. Massa de matéria seca acumulada na parte aérea (MMS), em razão da radiação fotossinteticamente ativa interceptada acumulada $\left(\mathrm{RFA}_{\text {int }}\right)$ durante o primeiro e segundo ciclos do cultivo do tomateiro, nos ambientes em estufa com tela $(\boldsymbol{\nabla})$, estufa sem tela (O) e externo à estufa (•). Eldorado do Sul, RS, 1999/2000. 


\section{Conclusões}

1. Para um mesmo valor de radiação fotossinteticamente ativa interceptada, se obtém maior eficiência de uso da radiação (matéria seca aérea acumulada) pelo tomateiro quando cultivado em estufa do que a céu aberto.

2. A eficiência de uso da radiação fotossinteticamente ativa pelo tomateiro foi maior no período verão-outono, época em que houve menor disponibilidade de radiação fotossinteticamente ativa incidente.

\section{Referências}

AIKMAN, D. P. Potential increase in photosynthetic efficiency from the redistribution of solar radiation in a crop. Journal of Experimental Botany, Oxford, v. 40, n. 217, p. 855-864, 1989.

ANDRIOLO, J. L.; FALCÃO, L. L. Efeito da poda de folhas sobre a acumulação de matéria seca e sua repartição para os frutos de tomateiro cultivado em ambiente protegido. Revista Brasileira de Agrometeorologia, Santa Maria, v. 8, n. 1, p. 75-83, 2000.

BOARDMAN, N. K. Comparative photosynthesis of sun and shade plants. Annual Review of Plant Physiology and Plant Molecular Biology, Palo Alto, v. 28, p. 355-377, 1977.

CAMACHO, M. J.; ASSIS, F. N.; MARTINS, S. R.; MENDEZ, M. E. G. Avaliação de elementos meteorológicos em estufa plástica em Pelotas, RS. Revista Brasileira de Agrometeorologia, Santa Maria, v. 3, p. 19-24, 1995.

COSTA, L. C.; MORISON, J. I. L.; DENNETT, M. D. Carbon balance of growing faba bean and its effect on crop growth: experimental and modeling approaches. Revista Brasileira de Agrometeorologia, Santa Maria, v. 4, p. 11-17, 1996.

FARIAS, J. R. B.; BERGAMASCHI, H.; MARTINS, S. R.; BERLATO, M. A. Efeito da cobertura plástica sobre a radiação solar. Revista Brasileira de Agrometeorologia, Santa Maria, v. 1, n. 1, p. 31-36, 1993.

GALLO, K. P.; DAUGHTRY, C. S. T.; WIEEGAND, C. L. Errors in measuring absorbed and computing crop radiation use efficiency. Agronomy Journal, Madison, v. 85, p. 1222-1228, 1993.

GISLEROD, H. R.; MORTENSEN, L. M. Air humidity and nutrient concentration affect nutrient uptake and growth of some greenhouse plants. Acta Horticulturae, Wageningen, n. 294, p. 141-146, 1991.
HAMMER, G. L.; VANDERLIP, R. L. Genotypeby-environment interaction in grain sorghum - I: effects of temperature on radiation use efficiency. Crop Science, Madison, v. 29, p. 370-376, 1989.

HEUVELINK, E. Growth, development and yield of a tomato crop: periodic destructive measurements in a greenhouse. Scientia Horticulturae, Amsterdam, v. 61, p. 77-99, 1995.

MONTEITH, J. L. Climate and the efficiency of crop production in Britain. Proceedings of the Royal Society of London, London, v. 281, p. 277-294, 1977.

MÜLLER, A. G. Modelagem da matéria seca e do rendimento de grãos de milho em relação à disponibilidade hídrica. 2001. $120 \mathrm{f}$. Tese (Doutorado em Fitotecnia) - Universidade Federal do Rio Grande do Sul, Porto Alegre, 2001.

PAPADOPOULOS, A. P.; ORMROD, D. P. Plant spacing effects on light interception by greenhouse tomatoes. Canadian Journal of Plant Science, Ottawa, v. 68 , p. 1197-1208, 1988.

RUSSELL, G.; JARVIS, P. G.; MONTEITH, J. L. Absorption of radiation by canopies and stand growth. In: RUSSELL, G.; MARSHALL, B.; JARVIS, P. G. (Ed.). Plant canopies: their growth, form and function. Cambridge, Inglaterra: Cambridge University Press: 1989. p. 21-39. (Society for Experimental Biology Seminar Series, 31).

SINCLAIR, T. R.; HORIE, T. Leaf nitrogen, photosynthesis, and crop radiation use efficiency: a review. Crop Science, Madison, v. 29, p. 98-105, 1989.

SINCLAIR, T. R.; SHIRAIWA, T. Soybean radiation-use efficiency as influenced by nonuniform specific leaf nitrogen distribution radiation. Crop Science, Madison, v. 33 , p. 808-812, 1993.

SINCLAIR, T. R.; SHIRAIWA, T.; HAMMER, G. L. Variation in crop radiation-use efficiency with increased diffuse radiation. Crop Science, Madison, v. 32, p. 1281-1284, 1992

SIVAKUMAR, M. V. K.; VIRMANI, S. M. Crop productivity in relation to interception of photosynthetically active radiation. Agricultural and Forest Meteorology, Amsterdam, v. 31, p. 131-141, 1984.

THIMIJAN, R. W.; HEINS, R. D. Photometric, radiometric, and quantum light units of measure: a review of procedures for interconversion. HortScience, Alexandria, v. 18, n. 6, p. 818-822, 1983.

VARLET-GRANCHER, C.; GOSSE, G.; CHARTIER, M.; SINOQUET, H.; BONHOMME, R.; ALLIRAND, J. M. Mise au point: rayonnement solaire absorbé ou intercepté par un couvert végétal. Agronomie, Paris, v. 9, p. 419-439, 1989. 